\title{
Dynamically generated resonances from the vector octet-baryon octet interaction
}

\author{
E. Oset ${ }^{1}$ and A. Ramos ${ }^{2}$
}

March 3, 2010

${ }^{1}$ Departamento de Física Teórica and IFIC, Centro Mixto Universidad de Valencia-CSIC, Institutos de Investigación de Paterna, Aptdo. 22085, 46071 Valencia, Spain

2 Departament d'Estructura i Constituents de la Matèria and Institut de Ciències del Cosmos, Universitat de Barcelona, 08028 Barcelona, Spain

\begin{abstract}
We study the interaction of vector mesons with the octet of stable baryons in the framework of the local hidden gauge formalism using a coupled channels unitary approach. We examine the scattering amplitudes and their poles, which can be associated to known $J^{P}=1 / 2^{-}, 3 / 2^{-}$baryon resonances, in some cases, or give predictions in other ones. The formalism employed produces doublets of degenerate $J^{P}=1 / 2^{-}, 3 / 2^{-}$states, a pattern which is observed experimentally in several cases. The findings of this work should also be useful to guide present experimental programs searching for new resonances, in particular in the strange sector where the current information is very poor.
\end{abstract}

\section{Introduction}

The use of chiral Lagrangians in combination with unitary techniques in coupled channels of mesons and baryons has been a very fruitful scheme to study the nature of many hadron resonances. The analysis of meson baryon scattering amplitudes shows poles in the second Riemann sheet which are identified with existing baryon resonances. In this way the interaction of the octet of pseudoscalar mesons with the octet of stable baryons has lead to $J^{P}=1 / 2^{-}$resonances which fit quite well the spectrum of the known low lying resonances with these quantum numbers [1-9]. Similarly, the interaction of the octet of pseudoscalar mesons with the decuplet of baryons also leads to many resonances that can be identified with existing ones of $J^{P}=3 / 2^{-}[13,14]$. Sometimes a new resonance is predicted, as in the case of the $\Lambda(1405)$, where all the chiral approaches find two poles close by, rather than one, a fact that finds experimental support in the analyses of Refs. [11, 12]. The nature of the resonances is admittedly more complex than just a molecule of pseudoscalar 
and baryon, but the success of this picture in reproducing many experimental data on decay and production of the resonances provides support to claim very large components of this character for the resonance wave function. In some cases one can even reach the limits of the model and find observables that call for extra components, even if small, but essential to explain some data. This can be seen for instance in the radiative decay of the $\Lambda(1520)$ [10], or in the helicity form factors of the $N^{*}(1535)$ [15]. Another promising approach is the one followed in [16], based on the naturalness of the subtraction constants in the dispersion relations that hint in some cases at the existence of non meson baryon components, particularly in the case of the $N^{*}(1535)$. Another step forward in this direction has been the interpretation of low lying $J^{P}=1 / 2^{+}$as molecular states of two pseudoscalar mesons and one baryon [17-21].

Much work has been done using pseudoscalar mesons as building blocks, but the consideration of vectors instead of pseudoscalars is only beginning to be exploited. In the baryon sector the interaction of the $\rho \Delta$ has been recently addressed in [22], where three degenerate $N^{*}$ states around $1800 \mathrm{MeV}$ and three degenerate $\Delta$ states around $1900 \mathrm{MeV}$, with $J^{P}=1 / 2^{-}, 3 / 2^{-}, 5 / 2^{-}$, are found. This work has been recently extended to the $\mathrm{SU}(3)$ space of vectors and baryons of the decuplet in [23]. The underlying theory for this study is the hidden gauge formalism [24-27], which deals with the interaction of vector mesons and pseudoscalars in a way respecting chiral dynamics, providing the interaction of pseudoscalars among themselves, with vector mesons, and vector mesons among themselves. It also offers a perspective on the chiral Lagrangians as limiting cases at low energies of vector exchange diagrams occurring in the theory. In a more recent work, looking for poles in the $\pi N$ scattering amplitudes, the $\rho N$ channel is also included [28] and a resonance around $1700 \mathrm{MeV}$ is dynamically generated, having the strongest coupling to this later channel.

In the meson sector, the interaction of $\rho \rho$ within this formalism has been addressed in [29], where it has been shown to lead to the dynamical generation of the $f_{2}(1270)$ and $f_{0}(1370)$ meson resonances, with a branching ratio for the sensitive $\gamma \gamma$ decay channel in good agreement with experiment [30]. This work has been extended to the interaction of the $\mathrm{SU}(3)$ vector mesons in [31], where several known resonances are also dynamically generated.

In the present work we study the interaction of the octet of vector mesons with the octet of stable baryons, using the unitary approach in coupled channels. We shall see that the scattering amplitudes lead to poles in the complex plane which can be associated to some well known resonances. Under the approximation of neglecting the three momentum of the particles versus their mass, we obtain degenerate states of $J^{P}=1 / 2^{-}, 3 / 2^{-}$, a pattern which seems to be followed qualitatively by the experimental spectrum, although in some cases the spin partners have not been identified. A different approach to account for the vector-baryon interaction is the one followed in [32] where pseudoscalar mesons, vector mesons and baryons mix, advocating SU(6) symmetry for the interaction. The approach leads to the same pseudoscalar-baryon interaction than the hidden gauge approach, but to different results when it comes to the interaction of the vector mesons with baryons. In particular, the spin degeneracy predicted by the hidden gauge approach does not show up in the matrix elements of the potential in the $\mathrm{SU}(6)$ scheme. 


\section{Formalism for $V V$ interaction}

We follow the formalism of the hidden gauge interaction for vector mesons of [24-27] (see also [33] for a practical set of Feynman rules). The Lagrangian involving the interaction of vector mesons amongst themselves is given by

$$
\mathcal{L}_{I I I}=-\frac{1}{4}\left\langle V_{\mu \nu} V^{\mu \nu}\right\rangle
$$

where the symbol \langle\rangle stands for the trace in the $S U(3)$ space and $V_{\mu \nu}$ is given by

$$
V_{\mu \nu}=\partial_{\mu} V_{\nu}-\partial_{\nu} V_{\mu}-i g\left[V_{\mu}, V_{\nu}\right]
$$

where $g$ is

$$
g=\frac{M_{V}}{2 f},
$$

with $f=93 \mathrm{MeV}$ the pion decay constant. With the value of $g$ of eq. (3) one fulfills the KSFR rule [34] which is tied to vector meson dominance [35]. The magnitude $V_{\mu}$ is the $S U(3)$ matrix of the vectors of the octet of the $\rho$

$$
V_{\mu}=\left(\begin{array}{ccc}
\frac{\rho^{0}}{\sqrt{2}}+\frac{\omega}{\sqrt{2}} & \rho^{+} & K^{*+} \\
\rho^{-} & -\frac{\rho^{0}}{\sqrt{2}}+\frac{\omega}{\sqrt{2}} & K^{* 0} \\
K^{*-} & \bar{K}^{* 0} & \phi
\end{array}\right)_{\mu}
$$

The lagrangian $\mathcal{L}_{I I I}$ gives rise to a contact term coming from $\left[V_{\mu}, V_{\nu}\right]\left[V_{\mu}, V_{\nu}\right]$

$$
\mathcal{L}_{I I I}^{(c)}=\frac{g^{2}}{2}\left\langle V_{\mu} V_{\nu} V^{\mu} V^{\nu}-V_{\nu} V_{\mu} V^{\mu} V^{\nu}\right\rangle
$$

as well as to a three vector vertex from

$$
\mathcal{L}_{I I I}^{(3 V)}=i g\left\langle\left(\partial_{\mu} V_{\nu}-\partial_{\nu} V_{\mu}\right) V^{\mu} V^{\nu}\right\rangle
$$

It is convenient to rewrite the Lagrangian of eq. (6) as

$$
\begin{aligned}
\mathcal{L}_{I I I}^{(3 V)} & =i g\left\langle V^{\nu} \partial_{\mu} V_{\nu} V^{\mu}-\partial_{\nu} V_{\mu} V^{\mu} V^{\nu}\right\rangle \\
& =i g\left\langle V^{\mu} \partial_{\nu} V_{\mu} V^{\nu}-\partial_{\nu} V_{\mu} V^{\mu} V^{\nu}\right\rangle \\
& =i g\left\langle\left(V^{\mu} \partial_{\nu} V_{\mu}-\partial_{\nu} V_{\mu} V^{\mu}\right) V^{\nu}\right\rangle .
\end{aligned}
$$

In this case one finds an analogy to the coupling of vectors to pseudoscalars given in the same theory by

$$
\mathcal{L}_{V P P}=-i g\left\langle\left[P, \partial_{\nu} P\right] V^{\nu}\right\rangle,
$$

where $P$ is the $\mathrm{SU}(3)$ matrix of the pseudoscalar fields. 
In a similar way, one obtains the Lagrangian for the coupling of vector mesons to the baryon octet given by $[36,37]^{1}$

$$
\mathcal{L}_{B B V}=g\left(\left\langle\bar{B} \gamma_{\mu}\left[V^{\mu}, B\right]\right\rangle+\left\langle\bar{B} \gamma_{\mu} B\right\rangle\left\langle V^{\mu}\right\rangle\right)
$$

where $B$ is now the $\mathrm{SU}(3)$ matrix of the baryon octet

$$
B=\left(\begin{array}{ccc}
\frac{1}{\sqrt{2}} \Sigma^{0}+\frac{1}{\sqrt{6}} \Lambda & \Sigma^{+} & p \\
\Sigma^{-} & -\frac{1}{\sqrt{2}} \Sigma^{0}+\frac{1}{\sqrt{6}} \Lambda & n \\
\Xi^{-} & \Xi^{0} & -\frac{2}{\sqrt{6}} \Lambda
\end{array}\right)
$$

With these ingredients we can construct the Feynman diagrams that lead to the $P B \rightarrow$ $P B$ and $V B \rightarrow V B$ interaction, by exchanging a vector meson between the pseudoscalar or the vector meson and the baryon, as depicted in Fig. 1.

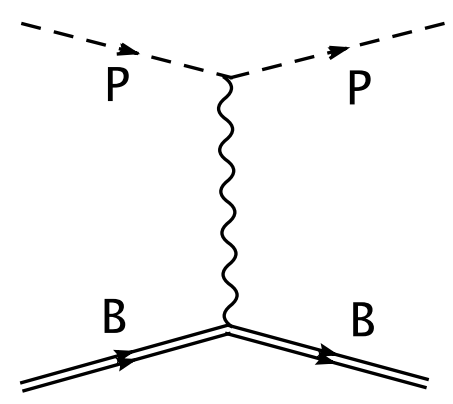

(a)

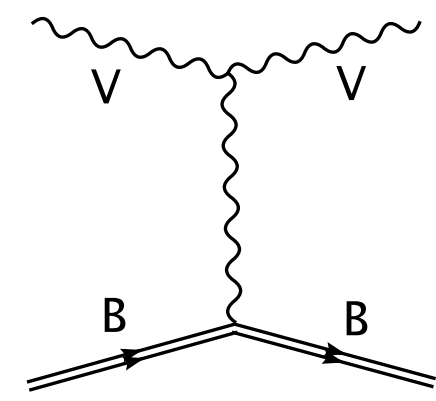

(b)

Figure 1: Diagrams contributing to the pseudoscalar-baryon (a) or vector- baryon (b) interaction via the exchange of a vector meson.

From the diagram of Fig. 1(a), and under the low energy approximation of neglecting $q^{2} / M_{V}^{2}$ in the propagator of the exchanged vector, where $q$ is the momentum transfer, one obtains the same amplitudes as obtained from the ordinary chiral Lagrangian for pseudoscalar-baryon octet interaction $[38,39]$, namely the Weinberg-Tomozawa terms. One could anticipate some analogy between the vector-baryon amplitudes with the pseudoscalarbaryon ones, given the similarity of the Lagrangians in the way we have written them in Eqs. (7) and (8). However, one also anticipates differences. Indeed, in the case of the pseudoscalar, Fig. 1(a), there is only one vector meson in the PPV coupling and this must necessarily be the one that is exchanged in the diagram. However, in the vector case, Fig. 1(b), the $V V V$ vertex involves three vector mesons and any of them can correspond in principle to the exchanged vector in the diagram. Nevertheless, one can proceed consequently with neglecting $q^{2} / M_{V}^{2}$ as implicit in the chiral Lagrangians [38,39], by also

\footnotetext{
${ }^{1}$ Correcting a misprint in $[36]$
} 
neglecting the three momentum of the external vectors versus the vector mass. In this case, the polarization vectors of the external vector mesons have only spatial components, since the zeroth component is either zero for the transverse polarizations, or negligible for the longitudinal one, $(k / \omega(k))$. Then, by examining the Lagrangian of Eq. (7) one realizes that the field $V^{\nu}$ cannot correspond to an external vector meson. Indeed, if this were the case, the $\nu$ index would be spatial and then the partial derivative $\partial_{\nu}$ would lead to a three momentum of the vector mesons which are neglected in the approach. We can then conclude that the field $V^{\nu}$ corresponds to the exchanged vector and the analogy with the pseudoscalar and vector interaction is then evident. Indeed, they are formally identical, by substituting the octet of pseudoscalar fields by the octet of the vector fields, with the additional factor $\vec{\epsilon} \vec{\epsilon}^{\prime}$ in the case of the interaction of the vector mesons. Note that $\epsilon_{\mu} \epsilon^{\mu}$ becomes $-\vec{\epsilon} \vec{\epsilon}^{\prime}$ and the signs of the Lagrangians also agree.

A small amendment is in order in the case of vector mesons, which is due to the mixing of $\omega_{8}$ and the singlet of $\mathrm{SU}(3), \omega_{1}$, to give the physical states of the $\omega$ and the $\phi$ mesons:

$$
\begin{aligned}
& \omega=\sqrt{\frac{2}{3}} \omega_{1}+\frac{1}{\sqrt{3}} \omega_{8} \\
& \phi=\frac{1}{\sqrt{3}} \omega_{1}-\sqrt{\frac{2}{3}} \omega_{8}
\end{aligned}
$$

Given the structure of Eq. (11), the singlet state which is accounted for by the V matrix, $\operatorname{diag}\left(\omega_{1}, \omega_{1}, \omega_{1}\right) / \sqrt{3}$, does not provide any contribution to Eq. (7), in which case all one must do is to take the matrix elements known for the $P B$ interaction and, wherever $P$ corresponds to the $\eta_{8}$, the amplitude should be multiplied by the factor $1 / \sqrt{3}$ to get the corresponding $\omega$ contribution, and by $-\sqrt{2 / 3}$ to get the corresponding $\phi$ contribution. Upon the approximation consistent with neglecting the three momentum versus the mass of the particles (in this case the baryon), we can just take the $\gamma^{0}$ component of Eq. (9) and then the transition potential corresponding to the diagram of $1(\mathrm{~b})$ is given by

$$
V_{i j}=-C_{i j} \frac{1}{4 f^{2}}\left(k^{0}+k^{\prime 0}\right) \vec{\epsilon} \vec{\epsilon}^{\prime}
$$

where $k^{0}, k^{\prime 0}$ are the energies of the incoming and outgoing vector meson.

The $C_{i j}$ coefficients of eq. (12) can be obtained directly from $[4,40,41]$ with the simple rules given above for the $\omega$ and the $\phi$ mesons, and substituting $\pi$ by $\rho$ and $K$ by $K^{*}$ in the matrix elements. The coefficients are obtained both in the physical basis of states or in the isospin basis. Here we will directly study the interaction in isospin basis and in the appendix we collect the tables of the $C_{i j}$ coefficients for different states of isospin, $I$, and strangeness, $S$. The tables immediately show the sectors where there is attraction and therefore give chances to find bound states or resonances. We can see that the cases with $(I, S)=(3 / 2,0)$, $(2,-1),(3 / 2,-2),(0,-3)$ and $(1,-3)$, the last four corresponding to exotic channels, are either zero or repulsive and do not produce poles in the scattering matrices. However, the sectors $(I, S)=(1 / 2,0),(0,-1),(1,-1)$ and $(1 / 2,-2)$ are attractive and we expect to find bound states and resonances in these cases. 
The next step to construct the scattering matrix implies solving the coupled channels Bethe Salpeter equation in the on shell factorization approach of $[4,5]$

$$
T=[1-V G]^{-1} V
$$

with $G$ being the loop function of a vector meson and a baryon which we calculate in dimensional regularization using the formula of [5]

$$
\begin{aligned}
G_{l}=i 2 M_{l} \int \frac{d^{4} q}{(2 \pi)^{4}} \frac{1}{(P-q)^{2}-M_{l}^{2}+i \epsilon} \frac{1}{q^{2}-m_{l}^{2}+i \epsilon} \\
=\frac{2 M_{l}}{16 \pi^{2}}\left\{a_{l}(\mu)+\ln \frac{M_{l}^{2}}{\mu^{2}}+\frac{m_{l}^{2}-M_{l}^{2}+s}{2 s} \ln \frac{m_{l}^{2}}{M_{l}^{2}}+\right. \\
\quad+\frac{\bar{q}_{l}}{\sqrt{s}}\left[\ln \left(s-\left(M_{l}^{2}-m_{l}^{2}\right)+2 \bar{q}_{l} \sqrt{s}\right)+\ln \left(s+\left(M_{l}^{2}-m_{l}^{2}\right)+2 \bar{q}_{l} \sqrt{s}\right)\right. \\
\left.\left.\quad-\ln \left(-s+\left(M_{l}^{2}-m_{l}^{2}\right)+2 \bar{q}_{l} \sqrt{s}\right)-\ln \left(-s-\left(M_{l}^{2}-m_{l}^{2}\right)+2 \bar{q}_{l} \sqrt{s}\right)\right]\right\}
\end{aligned}
$$

with $\mu$ a regularization scale, which we take to be $630 \mathrm{MeV}$, and with a natural value of the subtraction constant $a_{l}(\mu)$ of -2 , as determined in [5].

The iteration of diagrams implicit in the Bethe Salpeter equation in the case of the vector mesons has a subtlety with respect to the case of the pseudoscalars. The $\vec{\epsilon} \vec{\epsilon}^{\prime}$ term of the interaction forces the intermediate vector mesons in the loops to propagate with the spatial components in the loops. We need to sum over the polarizations of the internal vector mesons which, because they are tied to the external ones through the $\vec{\epsilon} \vec{\epsilon}^{\prime}$ factor, provides

$$
\sum_{p o l} \epsilon_{i} \epsilon_{j}=\delta_{i j}+\frac{q_{i} q_{j}}{M_{V}^{2}}
$$

As shown in [43], the on shell factorization leads to a correction coefficient in the $G$ function of $\vec{q}^{2} / 3 M_{V}^{2}$ versus unity, which is negligibly small, and which we also neglect here in consonance with the approximations done. In this case the factor $\vec{\epsilon} \vec{\epsilon}^{\prime}$, appearing in the potential $V$, factorizes also in the $T$ matrix for the external vector mesons. More especifically, the on-shell factorization is obtained in the following way. First, as mentioned above, the vector propagator in the vector exchange diagram that provides the potential is taken as $-1 / M_{V}^{2}$, leading to a three-momentum independent expression [see Eq. (12)], which can be factorized out of the $d^{3} k^{\prime \prime}$ integral in the loops. When the $d k^{0 \prime \prime}$ integral is performed, the value of $k^{0 \prime \prime}$ in Eq. (12) becomes the on shell variable $k^{0 \prime}$ as shown explicitly in Ref. [4], and thus the potential kernel, evaluated with the on shell values of $k^{0}$ and $k^{0 \prime}$ for a given value of the total energy $\sqrt{s}$, factorizes completely out of the four-dimensional $d^{4} k^{\prime \prime}$ integration.

One can obtain a feeling on how good the approximation of neglecting the term $q^{2} / M_{V}^{2}$ in the propagator of the exchanged vector is, from the following discussion. As shown in Sect. 2.2 of Ref. [23], the ratio of the s-wave projected $\rho$ propagator keeping the $q^{2} / m_{\rho}^{2}$ term to the one with this term neglected is given by

$$
R=\frac{m_{\rho}^{2}}{4|\vec{k}|^{2}} \ln \frac{m_{\rho}^{2}+4|\vec{k}|^{2}}{m_{\rho}^{2}},
$$


where $\vec{k}$ is the on-shell momentum of the incoming meson. For a given center-of-mass energy, the diversion from unity of this ratio is larger for the scattering of vector mesons than for pseudoscalars. Yet, in the case of $\rho N$ scattering, $R$ is within $10 \%$ for energies of the $\rho N$ system lying $25 \mathrm{MeV}$ above or below threshold. Even then, as shown in [23], a better agreement of the two prescriptions is possible with a slight change in the subtraction constant of the $\mathrm{G}$ function.

A detailed discussion of the factorization procedure for the particular case of vector mesons, after consideration of the $t$ - and $u$-channel analytical cuts can also be found in Sect. 2.2 of Ref. [23].

\section{Convolution due to the $\rho$ and $K^{*}$ mass distributions}

The formalism described above would provide results obtained using fixed masses for the vector mesons and no width. The mass distributions of the $\rho$ and $K^{*}$ mesons are sufficiently extended to advise a more accurate calculation that takes this large width into account. We follow the traditional method of convoluting the $G$ function with the mass distributions of the $\rho$ or $K^{*}$ mesons, as is customarily made [42]. One can prove that this convolution is equivalent to calculating the loop function with the dressed vector meson propagator written in terms of its Lehmann representation, as is done in calculations of medium effects in the scattering matrices [44]. The method amounts to replacing the $G$ function by $\tilde{G}$ obtained as

$$
\tilde{G}(s)=\frac{1}{N} \int_{\left(m-2 \Gamma_{i}\right)^{2}}^{\left(m+2 \Gamma_{i}\right)^{2}} d \tilde{m}^{2}\left(-\frac{1}{\pi}\right) \operatorname{Im} \frac{1}{\tilde{m}^{2}-m^{2}+\mathrm{i} \tilde{m} \Gamma(\tilde{m})} \quad G\left(s, \tilde{m}^{2}, \tilde{M}_{B}^{2}\right),
$$

with

$$
N=\int_{\left(m_{\rho}-2 \Gamma_{i}\right)^{2}}^{\left(m_{\rho}+2 \Gamma_{i}\right)^{2}} d \tilde{m}^{2}\left(-\frac{1}{\pi}\right) \operatorname{Im} \frac{1}{\tilde{m}^{2}-m_{\rho}^{2}+\mathrm{i} \tilde{m} \Gamma(\tilde{m})}
$$

being the normalization factor, and $\Gamma_{i}$ the decay width of the meson $\left(i=\rho, K^{*}\right)$, which we take to be $149.4 \mathrm{MeV}$ and $50.5 \mathrm{MeV}$ for the $\rho$ and $K^{*}$ meson, respectively. The energy dependent width $\Gamma(\tilde{m})$ for the $\rho$ meson, obtained from its decay into two pions in $p$-wave, is given by

$$
\Gamma(\tilde{m})=\Gamma_{\rho} \frac{m_{\rho}^{2}}{\tilde{m}^{2}}\left(\frac{\tilde{m}^{2}-4 m_{\pi}^{2}}{m_{\rho}^{2}-4 m_{\pi}^{2}}\right)^{3 / 2} \theta\left(\tilde{m}-2 m_{\pi}\right) .
$$

A similar expression gives the energy dependent width of the $K^{*}$ meson from its decay into a $K$ meson and a pion.

We will see that, using fixed masses for the vector mesons, one finds bound states in the $\rho N$ and $K^{*} N$ amplitudes, i.e. states having zero width. However, when $\tilde{G}$ is used in Eq. (13) and, therefore, both the $\rho$ and $K^{*}$ vector mesons are taken with their corresponding mass distribution, there is phase space for the decay of each of these bound states into some of the mass components of the vector meson and the nucleon, thereby acquiring an appreciable width. 


\section{Search for poles}

We search for poles in the scattering matrices in the second Riemann sheet, as defined in previous works [43], basically changing $\bar{q}_{l}$ by to $-\bar{q}_{l}$ in the analytical formula of the $G$ function, Eq. (14), for channels where $\operatorname{Re}(\sqrt{s})$ is above the threshold of the corresponding channel. When one has a mass distribution of the $\rho$ and $K^{*}$ mesons, and hence a fuzzy description of the threshold for some channels, one could take different prescriptions for going to the optimal Riemann sheet that better reflects the behavior of the amplitude in the real axis, which is where the physical information is contained. The results are very similar in all cases, expect when one has a resonance very close to threshold, where the convolution can distort the shape of the amplitude and even make the pole disappear. In view of that, for these cases the couplings are obtained from the amplitudes in the real axis as follows. Assuming these amplitudes to behave as

$$
T_{i j}=\frac{g_{i} g_{j}}{\sqrt{s}-M_{R}+i \Gamma / 2},
$$

where $M_{R}$ is the position of the maximum of $\left|T_{i i}\right|$, with $i$ being the channel to which the resonance couples more strongly, and $\Gamma$ its width at half-maximum, one then finds

$$
\left|g_{i}\right|^{2}=\frac{\Gamma}{2} \sqrt{\left|T_{i i}\right|^{2}}
$$

Up to a global phase, this expression allows one to determine the value of $g_{i}$, which we take to be real. The other couplings are then derived from

$$
g_{j}=g_{i} \frac{T_{i j}\left(\sqrt{s}=M_{R}\right)}{T_{i i}\left(\sqrt{s}=M_{R}\right)} .
$$

This procedure to obtain the couplings from $|T|^{2}$ in the real axis was used in [30] where it was found that changes in the input parameters that lead to moderate changes in the position and the width of the states affected the couplings more smoothly. Having this in mind, we also calculate, as a check, the couplings in the case of the particles without width from the residues of the amplitudes in the first Riemann sheet of the complex plane. The couplings obtained in this way are very similar to those obtained by means of Eqs. (21) and (22) employing amplitudes in the real axis obtained with the convolution method. The differences between the couplings obtained with the two methods are of the order of $10-20 \%$.

\section{Results}

In this section we show our results obtained in the attractive sectors mentioned above.

In Fig. 2 we show the results of $\left|T_{i i}\right|^{2}$ as a function of $\sqrt{s}$ for the different channels in the $(I, S)=(1 / 2,0)$ sector. We can see a neat bump for $\rho N \rightarrow \rho N$ around $1700 \mathrm{MeV}$, a few MeV below the $\rho N$ threshold. The width of the peak is due to the convolution of the $\rho$ 
mass. For a $\rho$ of fixed mass and no width one obtains a bound state with a small binding. The bump around $1700 \mathrm{MeV}$ is also seen in the $K^{*} \Lambda$ channel but is absent or barely visible in the $\omega N, \phi N$ and $K^{*} \Sigma$ channels. On the other hand, with the same strangeness and isospin, one finds a second peak around $1970 \mathrm{MeV}$, which is clearly visible in the $K^{*} \Lambda$, $\omega N, \phi N$ and $K^{*} \Sigma$ channels but not visible in the $\rho N$ one. This special behavior has to do with the coupling of the resonances to the different channels which will be studied below.

In Fig. 3 we show $\left|T_{i i}\right|^{2}$ for two cases, $(I, S)=(0,-1)$ and $(1,-1)$. For $I=0$ we see again a clear peak behavior of $\left|T_{i i}\right|^{2}$ around $1800 \mathrm{MeV}$ in the $\bar{K}^{*} N$ channel. The structure is also seen in all the other channels, except in the $K^{*} \Xi$ one. In the $\rho \Sigma$ channel one can see another structure around $1920 \mathrm{MeV}$, below the $\rho \Sigma$ threshold, which could be associated to a bound state of this system. Finally in the $K^{*} \Xi$ channel one finds another clear structure corresponding to a resonance around $2140 \mathrm{MeV}$, practically invisible in the other channels, which could qualify as a $K^{*} \Xi$ bound state.

The $(I, S)=(1,-1)$ case shows a clear peak around $1830 \mathrm{MeV}$ in the $\bar{K}^{*} N$ channel. This structure is also seen in other channels, but it could be the cusp due to the opening of the $\bar{K}^{*} N$ channel. We will come back to this when we make a search for the poles of the $T$ matrix. In the $\rho \Sigma$ channel, and also visible in the $K^{*} \Xi$ channel, we see another less pronounced structure around $1990 \mathrm{MeV}$.

In Fig. 4 we display the sector $(I, S)=(1 / 2,-2)$. In this case we find a clear structure of resonant type around $2030 \mathrm{MeV}$ which is visible in the $\rho \Xi$ and $\bar{K}^{*} \Lambda$ channels. Another clear peak appears around $2080 \mathrm{MeV}$, shortly below the $\bar{K}^{*} \Sigma$ threshold, which is more pronounced in the $\bar{K}^{*} \Sigma$ channel and appears as an interference minimum in the $\bar{K}^{*} \Lambda$ channel.

Since the spin dependence only comes from the $\vec{\epsilon} \vec{\epsilon}^{\prime}$ factor and there is no dependence on the spin of the baryons, the interaction for vector-baryon states with $1 / 2^{-}$and $3 / 2^{-}$ is the same and then one should associate each peak with the existence of two degenerate states. The spin degeneration also appears in some quarks models [45].

The couplings of the resonances to the different channels, obtained from the residues at the poles are shown in Tables 1 to 4 .

\begin{tabular}{c|cc|cc}
\hline$z_{R}$ & \multicolumn{2}{|c|}{$1696^{(*)}$} & \multicolumn{2}{c}{$1977+\mathrm{i} 53$} \\
\hline & $g_{i}$ & $\left|g_{i}\right|$ & $g_{i}$ & $\left|g_{i}\right|$ \\
\hline$\rho N(1710)$ & $3.2+\mathrm{i} 0$ & 3.2 & $-0.3-\mathrm{i} 0.5$ & 0.6 \\
$\omega N(1721)$ & $0.1+\mathrm{i} 0$ & 0.1 & $-1.1-\mathrm{i} 0.4$ & 1.2 \\
$\phi N(1958)$ & $-0.2+\mathrm{i} 0$ & 0.2 & $1.5+\mathrm{i} 0.6$ & 1.7 \\
$K^{*} \Lambda(2010)$ & $2.3+\mathrm{i} 0$ & 2.3 & $2.2-\mathrm{i} 0.9$ & 2.3 \\
$K^{*} \Sigma(2087)$ & $-0.6+\mathrm{i} 0$ & 0.6 & $3.9+\mathrm{i} 0.2$ & 3.9 \\
\hline
\end{tabular}

Table 1: Pole position and coupling constants to various channels of the resonances found in the $I=1 / 2, S=0$ sector. ${ }^{(*)}$ The properties of this state have been determined from the amplitudes in the real axis, as explained in the text. 

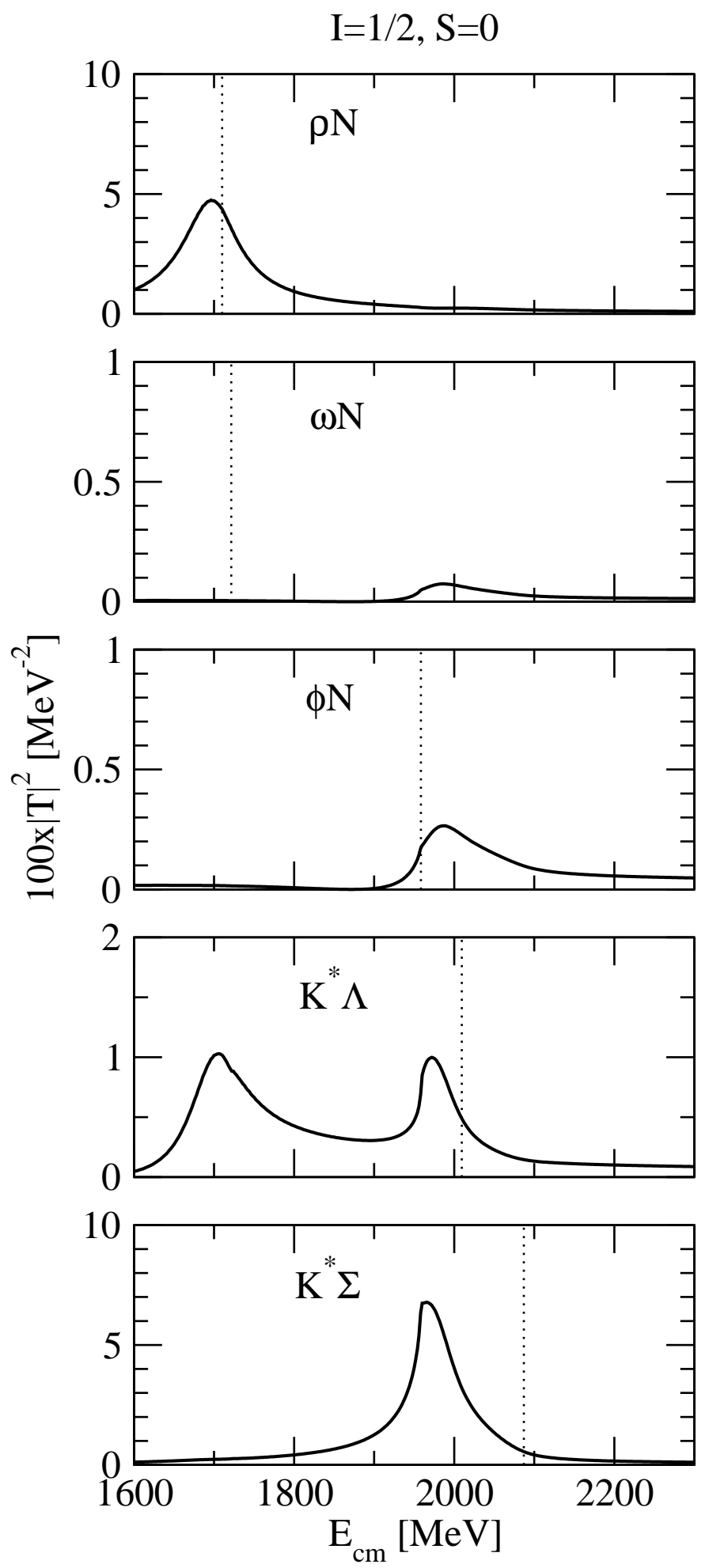

Figure 2: $|T|^{2}$ for different channels for $I=1 / 2$ and strangeness $S=0$. Channel thresholds are indicated by vertical dotted lines. 

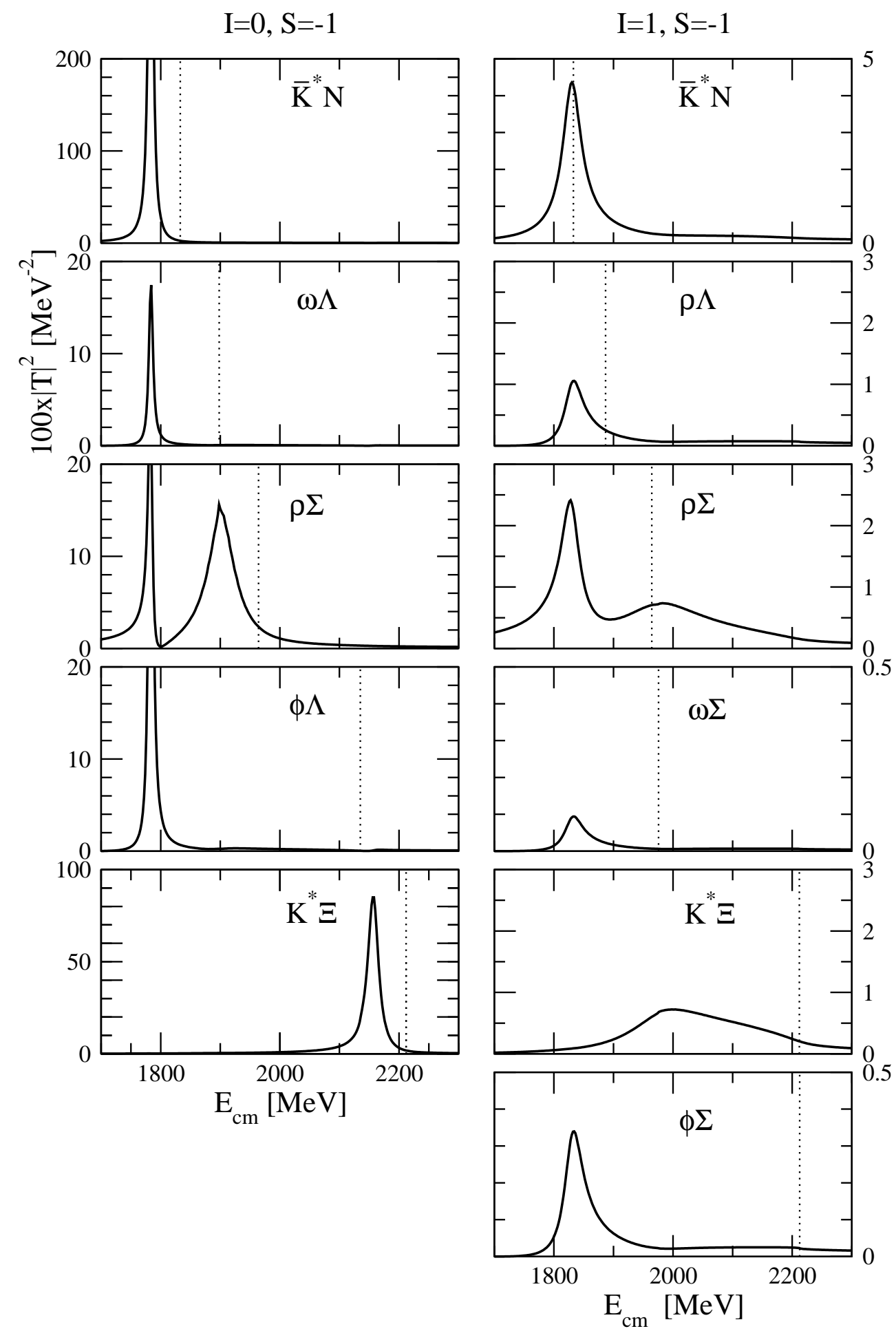

Figure 3: $|T|^{2}$ for different channels for $I=0,1$ and strangeness $S=-1$. Channel thresholds are indicated by vertical dotted lines. 


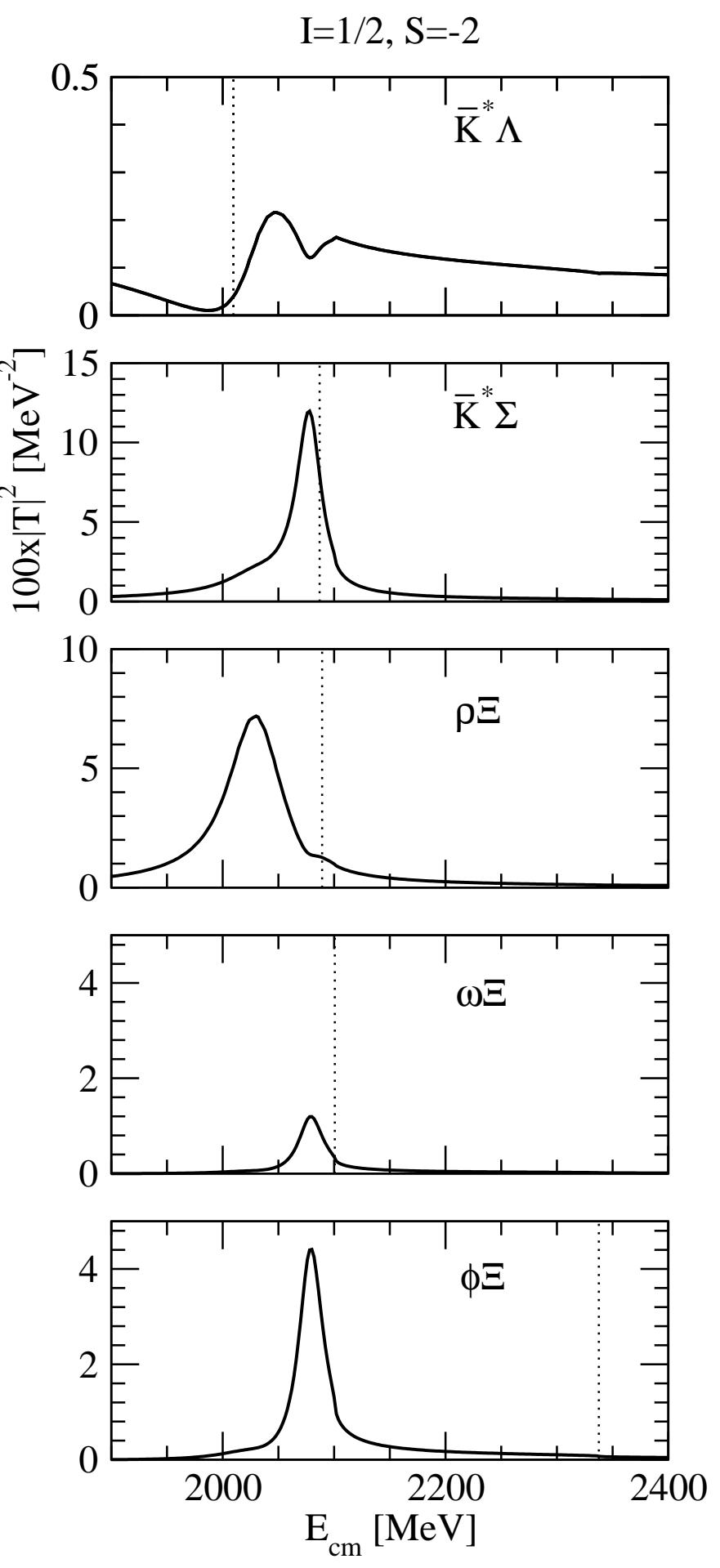

Figure 4: $|T|^{2}$ for different channels for $I=1 / 2$ and strangeness $S=-2$. Channel thresholds are indicated by vertical dotted lines. 


\begin{tabular}{c|cc|cc|cc}
\hline$z_{R}$ & \multicolumn{2}{|c|}{$1784+\mathrm{i} 4$} & \multicolumn{2}{c|}{$1906+\mathrm{i} 70$} & \multicolumn{2}{c}{$2158+\mathrm{i} 13$} \\
\hline & $g_{i}$ & $\left|g_{i}\right|$ & $g_{i}$ & $\left|g_{i}\right|$ & $g_{i}$ & $\left|g_{i}\right|$ \\
\hline $\bar{K}^{*} N(1833)$ & $3.3+\mathrm{i} 0.07$ & 3.3 & $0.1+\mathrm{i} 0.2$ & 0.3 & $0.2+\mathrm{i} 0.3$ & 0.3 \\
$\omega \Lambda(1898)$ & $1.4+\mathrm{i} 0.03$ & 1.4 & $0.4+\mathrm{i} 0.2$ & 0.5 & $-0.3-\mathrm{i} 0.2$ & 0.4 \\
$\rho \Sigma(1964)$ & $-1.5+\mathrm{i} 0.03$ & 1.5 & $3.1+\mathrm{i} 0.7$ & 3.2 & $0.01-\mathrm{i} 0.08$ & 0.08 \\
$\phi \Lambda(2135)$ & $-1.9-\mathrm{i} 0.04$ & 1.9 & $-0.6-\mathrm{i} 0.3$ & 0.6 & $0.5+\mathrm{i} 0.3$ & 0.5 \\
$K^{*} \Xi(2212)$ & $0.1+\mathrm{i} 0.003$ & 0.1 & $0.3+\mathrm{i} 0.1$ & 0.3 & $3.2-\mathrm{i} 0.1$ & 3.2 \\
\hline
\end{tabular}

Table 2: Pole position and coupling constants to various channels of the resonances found in the $I=0, S=-1$ sector.

\begin{tabular}{c|cc|cc}
\hline$z_{R}$ & \multicolumn{2}{|c}{$1830^{(*)}$} & \multicolumn{2}{c}{$1987^{(*)}$} \\
\hline $\bar{K}^{*} N(1833)$ & $2.1+\mathrm{i} 0$ & 2.1 & $-0.3-\mathrm{i} 1.0$ & 1.0 \\
$\rho \Lambda(1887)$ & $-1.6+\mathrm{i} 0.2$ & 1.6 & $-0.3+\mathrm{i} 0.9$ & 0.9 \\
$\rho \Sigma(1964)$ & $-1.6+\mathrm{i} 0.07$ & 1.6 & $2.6+\mathrm{i} 0$ & 2.6 \\
$\omega \Sigma(1976)$ & $-0.9+\mathrm{i} 0.1$ & 0.9 & $-0.2+\mathrm{i} 0.5$ & 0.5 \\
$K^{*} \Xi(2212)$ & $0.1+\mathrm{i} 0.06$ & 0.1 & $2.1-\mathrm{i} 0.8$ & 2.3 \\
$\phi \Sigma(2213)$ & $1.2-\mathrm{i} 0.2$ & 1.2 & $0.2-\mathrm{i} 0.7$ & 0.7 \\
\hline
\end{tabular}

Table 3: Pole position and coupling constants to various channels of the resonances found in the $I=1, S=-1$ sector. ${ }^{(*)}$ The properties of this state have been determined from the amplitudes in the real axis, as explained in the text.

\begin{tabular}{c|cc|cc}
\hline$z_{R}$ & \multicolumn{2}{|c|}{$2039+\mathrm{i} 67$} & \multicolumn{2}{c}{$2082+\mathrm{i} 31$} \\
\hline & $g_{i}$ & $\left|g_{i}\right|$ & $g_{i}$ & $\left|g_{i}\right|$ \\
\hline $\bar{K}^{*} \Lambda(2010)$ & $-0.7-\mathrm{i} 0.5$ & 0.9 & $-0.1-\mathrm{i} 0.3$ & 0.4 \\
$\bar{K}^{*} \Sigma(2087)$ & $-0.9-\mathrm{i} 0.5$ & 1.0 & $1.8+\mathrm{i} 0.5$ & 1.9 \\
$\rho \Xi(2089)$ & $2.4+\mathrm{i} 0.7$ & 2.5 & $0.4+\mathrm{i} 0.3$ & 0.5 \\
$\omega \Xi(2101)$ & $0.6-\mathrm{i} 0.08$ & 0.6 & $1.1+\mathrm{i} 0.3$ & 1.2 \\
$\phi \Xi(2038)$ & $-0.8+\mathrm{i} 0.1$ & 0.8 & $-1.6-\mathrm{i} 0.4$ & 1.6 \\
\hline
\end{tabular}

Table 4: Pole position and coupling constants to various channels of the resonances found in the $I=1 / 2, S=-2$ sector.

\section{Comparison to data}

In table 5 we show a summary of the results obtained and the tentative association to known states [46]. 


\begin{tabular}{|c|c|c|c|c|c|c|c|c|}
\hline \multirow[t]{2}{*}{$I, S$} & \multicolumn{3}{|c|}{ Theory } & \multicolumn{5}{|c|}{ PDG data } \\
\hline & pole position & $\begin{array}{r}\text { rea } \\
\text { mass }\end{array}$ & $\begin{array}{l}\text { axis } \\
\text { width }\end{array}$ & name & $J^{P}$ & status & mass & width \\
\hline \multirow[t]{4}{*}{$1 / 2,0$} & - & 1696 & 92 & $N(1650)$ & $1 / 2^{-}$ & $\star \star \star \star$ & $1645-1670$ & $145-185$ \\
\hline & & & & $N(1700)$ & $3 / 2^{-}$ & $\star \star \star$ & $1650-1750$ & $50-150$ \\
\hline & $1977+\mathrm{i} 53$ & 1972 & 64 & $N(2080)$ & $3 / 2^{-}$ & $\star \star$ & $\approx 2080$ & $180-450$ \\
\hline & & & & $N(2090)$ & $1 / 2^{-}$ & 夫 & $\approx 2090$ & $100-400$ \\
\hline \multirow[t]{4}{*}{$0,-1$} & $1784+\mathrm{i} 4$ & 1783 & 9 & $\Lambda(1690)$ & $3 / 2^{-}$ & $\star \star \star \star ~$ & $1685-1695$ & $50-70$ \\
\hline & & & & $\Lambda(1800)$ & $1 / 2^{-}$ & $\star \star \star$ & $1720-1850$ & $200-400$ \\
\hline & $1907+\mathrm{i} 70$ & 1900 & 54 & $\Lambda(2000)$ & $?$ & $\star$ & $\approx 2000$ & $73-240$ \\
\hline & $2158+\mathrm{i} 13$ & 2158 & 23 & & & & & \\
\hline \multirow[t]{3}{*}{$1,-1$} & - & 1830 & 42 & $\Sigma(1750)$ & $1 / 2^{-}$ & $\star \star \star$ & $1730-1800$ & $60-160$ \\
\hline & - & 1987 & 240 & $\Sigma(1940)$ & $3 / 2^{-}$ & $\star \star \star$ & 1900-1950 & $150-300$ \\
\hline & & & & $\Sigma(2000)$ & $1 / 2^{-}$ & $\star$ & $\approx 2000$ & $100-450$ \\
\hline \multirow[t]{2}{*}{$1 / 2,-2$} & $2039+\mathrm{i} 67$ & 2039 & 64 & $\Xi(1950)$ & $?$ & $\star \star \star$ & $1950 \pm 15$ & $60 \pm 20$ \\
\hline & $2083+\mathrm{i} 31$ & 2077 & 29 & $\Xi(2120)$ & $?$ & 夫 & $\approx 2120$ & 25 \\
\hline
\end{tabular}

Table 5: The properties of the 9 dynamically generated resonances and their possible PDG counterparts.

For the $(I, S)=(1 / 2,0) N^{*}$ states there is the $N^{*}(1700)$ with $J^{P}=3 / 2^{-}$, which could correspond to the state we find with the same quantum numbers around the same energy. We also find in the PDG the $N^{*}(1650)$, which could be the near degenerate spin parter of the $N^{*}(1700)$ that we predict in the theory. The difference of $50 \mathrm{MeV}$, is also the typical difference found in the $\Delta(1900)\left(1 / 2^{-}\right), \Delta(1930)\left(5 / 2^{-}\right), \Delta(1940)\left(3 / 2^{-}\right)$states which are predicted as degenerate in the study of the interaction of vector mesons with the baryons of the decuplet in $[22,23]$. It is interesting to recall that in the study of Ref. [28], done within the framework of the Juelich model [47], a pole is found around $1700 \mathrm{MeV}$, with the largest coupling to $\rho N$ states. The coupling found there is such that the strongest strength corresponds, by far, to the $L=0, J^{P}=3 / 2^{-}$channel [48], which corresponds to the channel obtained in our approach. However, the pole in Ref. [28] moves away from this position when the $N^{*}(1520)$ resonance is introduced as a genuine resonance in their model, due to the mechanism of pole repulsion discussed in Ref. [49].

Around $2000 \mathrm{MeV}$, where we find another $N^{*}$ resonance, there are the states $N^{*}(2080)$ 
and $N^{*}(2090)$, with $J^{P}=3 / 2^{-}$and $J^{P}=1 / 2^{-}$respectively, showing a good approximate spin degeneracy.

For the case $(I, S)=(0,-1)$ there is in the PDG one state, the $\Lambda(1800)$ with $J^{P}=$ $1 / 2^{-}$, remarkably close to the energy were we find a $\Lambda$ state. The spin parter with $J^{P}=$ $3 / 2^{-}$is either absent in the PDG, or corresponds to the $\Lambda(1690)$, although this implies a large breaking of the expected degeneracy. The state obtained around $1900 \mathrm{MeV}$ could correspond to the $\Lambda(2000)$ cataloged in the PDG with unknown spin and parity. On the other hand, one does not find in the PDG a resonance to associate with the $\Lambda$ state predicted around $2150 \mathrm{MeV}$.

The case of the $\Sigma$ states having $(I, S)=(1,-1)$ is rather interesting. The state that we find around $1830 \mathrm{MeV}$, could be associated to the $\Sigma(1750)$ with $J^{P}=1 / 2^{-}$. More interesting seems to be the case of the state obtained around $1990 \mathrm{MeV}$ that could be related to two PDG candidates, again nearly degenerate, the $\Sigma(1940)$ and the $\Sigma(2000)$, with spin and parity $J^{P}=3 / 2^{-}$and $J^{P}=1 / 2^{-}$respectively.

Finally, for the case of the cascade resonances, $(I, S)=(1 / 2,-2)$, we find two states, one around $2040 \mathrm{MeV}$ and the other one around $2080 \mathrm{MeV}$. There are two cascade states in the PDG around this energy region with spin parity unknown, the $\Xi(1950)$ and the $\Xi(2120)$. The relatively small widths obtained in each case and the agreement with the experimental ones would be an extra feature to support the association of our states to these resonances. Although the experimental knowledge of this sector is relatively poor, a program is presently running at Jefferson Lab to improve on this situation [50].

The agreement found in general is encouraging. One should stress that the measurements of the masses and widths in this energy region are not easy, as one can guess from the dispersion of the data obtained in different experiments. The fact that some of the states predicted (essentially the spin partners) are not found in the PDG, should not be seen as a negative result of the theory, but as a motivation for the search of new resonances. The theory tells us the origin of these states, as coming from the vector baryon interaction. This gives us a new information and, although the larger part of the width may come from pseudoscalar baryon decay, it is in the vector baryon channels that experimental efforts should be made to eventually find these states and confirm their vector-baryon nature. The devoted search of $\Xi$ resonances at Jefferson Lab $[50,51]$ should be most welcome in this context.

\section{Conclusions}

We have studied the interaction of mesons in the vector octet of the $\rho$ with baryons of the octet of the proton within the hidden gauge formalism of vector mesons, using a unitary framework in coupled channels.

We observe a rich structure in the vector-baryon scattering amplitudes which is associated to the presence of poles in the complex plane. This structure is clearly visible in the real axis as neat peaks of $|T|^{2}$ in different channels. We could associate many of the states predicted by the theory to known states in the PDG, thus providing a very different 
explanation for the nature of these states than the one given by quark models as simple $3 q$ states. One of the particular predictions of the theory is that, within the approximations done, one obtains degenerate pairs of particles in $J^{P}=1 / 2^{-}, 3 / 2^{-}$. This behavior seems well reproduced by many of the existing data, but in some cases the spin partners do not show up in the PDG. The reasonable results produced by the hidden gauge approach in this case, as well as in other cases $[22,23]$ should give a stimulus to search experimentally for the missing spin partners of the already observed states, as well as possible new ones.

\section{Acknowledgments}

We would like to thank J. Garzon for checking the formulas of the paper. This work is partly supported by the EU contract No. MRTN-CT-2006-035482 (FLAVIAnet), by the contracts FIS2006-03438 FIS2008-01661 from MICINN (Spain) and by the Generalitat de Catalunya contract 2005SGR-00343. We acknowledge the support of the European Community-Research Infrastructure Integrating Activity "Study of Strongly Interacting Matter" (HadronPhysics2, Grant Agreement n. 227431) under the Seventh Framework Programme of EU.

\section{A Coefficients of the $s$-wave tree level amplitudes}

This Appendix gives the coefficients $C_{i j}^{I S}$ of the $s$-wave tree level vector-baryon amplitudes of Eq. (12) for the various $I S$ sectors studied in this work.

Table 6: Coefficients $C_{i j}^{I S}$ for the sector $I=1 / 2, S=0$.

\begin{tabular}{l|ccccc} 
& $\rho N$ & $\omega N$ & $\phi N$ & $K^{*} \Lambda$ & $K^{*} \Sigma$ \\
\hline$\rho N$ & 2 & 0 & 0 & $\frac{3}{2}$ & $-\frac{1}{2}$ \\
$\omega N$ & & 0 & 0 & $-\frac{3}{2} \frac{1}{\sqrt{3}}$ & $-\frac{3}{2} \frac{1}{\sqrt{3}}$ \\
$\phi N$ & & & 0 & $-\frac{3}{2}\left(-\sqrt{\frac{2}{3}}\right)$ & $-\frac{3}{2}\left(-\sqrt{\frac{2}{3}}\right)$ \\
$K^{*} \Lambda$ & & & & 0 & 0 \\
$K^{*} \Sigma$ & & & & & 2
\end{tabular}

Table 7: Coefficients $C_{i j}^{I S}$ for the sector $I=3 / 2, S=0$.

\begin{tabular}{c|cc} 
& $\rho N$ & $K^{*} \Sigma$ \\
\hline$\rho N$ & -1 & -1 \\
$K^{*} \Sigma$ & & -1
\end{tabular}


Table 8: Coefficients $C_{i j}^{I S}$ for the sector $I=0, S=-1$.

\begin{tabular}{l|ccccc} 
& $\bar{K}^{*} N$ & $\omega \Lambda$ & $\rho \Sigma$ & $\phi \Lambda$ & $K^{*} \Xi$ \\
\hline $\bar{K}^{*} N$ & 3 & $\frac{3}{\sqrt{2}} \frac{1}{\sqrt{3}}$ & $-\sqrt{\frac{3}{2}}$ & $\frac{3}{\sqrt{2}}\left(-\sqrt{\frac{2}{3}}\right)$ & 0 \\
$\omega \Lambda$ & & 0 & 0 & 0 & $-\frac{3}{\sqrt{2}} \frac{1}{\sqrt{3}}$ \\
$\rho \Sigma$ & & & 4 & 0 & $\sqrt{\frac{3}{2}}$ \\
$\phi \Lambda$ & & & & 0 & $-\frac{3}{\sqrt{2}}\left(-\sqrt{\frac{2}{3}}\right)$ \\
$K^{*} \Xi$ & & & & & 3
\end{tabular}

Table 9: Coefficients $C_{i j}^{I S}$ for the sector $I=1, S=-1$.

\begin{tabular}{l|cccccc} 
& $\bar{K}^{*} N$ & $\rho \Lambda$ & $\rho \Sigma$ & $\omega \Sigma$ & $K^{*} \Xi$ & $\phi \Sigma$ \\
\hline $\bar{K}^{*} N$ & 1 & $-\sqrt{\frac{3}{2}}$ & -1 & $-\sqrt{\frac{3}{2}} \frac{1}{\sqrt{3}}$ & 0 & $-\sqrt{\frac{3}{2}}\left(-\sqrt{\frac{2}{3}}\right)$ \\
$\rho \Lambda$ & & 0 & 0 & 0 & $-\sqrt{\frac{3}{2}}$ & 0 \\
$\rho \Sigma$ & & & 2 & 0 & 1 & 0 \\
$\omega \Sigma$ & & & & 0 & $-\sqrt{\frac{3}{2}} \frac{1}{\sqrt{3}}$ & 0 \\
$K^{*} \Xi$ & & & & & 1 & $-\sqrt{\frac{3}{2}}\left(-\sqrt{\frac{2}{3}}\right)$ \\
$\phi \Sigma$ & & & & & & 0
\end{tabular}

Table 10: Coefficients $C_{i j}^{I S}$ for the sector $I=2, S=-1$.

$$
\begin{array}{c|c} 
& \rho \Sigma \\
\hline \rho \Sigma & -2
\end{array}
$$

Table 11: Coefficients $C_{i j}^{I S}$ for the sector $I=1 / 2, S=-2$.

\begin{tabular}{l|ccccc} 
& $\bar{K}^{*} \Lambda$ & $\bar{K}^{*} \Sigma$ & $\rho \Xi$ & $\omega \Xi$ & $\phi \Xi$ \\
\hline $\bar{K}^{*} \Lambda$ & 0 & 0 & $-\frac{3}{2}$ & $-\frac{3}{2} \frac{1}{\sqrt{3}}$ & $-\frac{3}{2}\left(-\sqrt{\frac{2}{3}}\right)$ \\
$\bar{K}^{*} \Sigma$ & & 2 & $-\frac{1}{2}$ & $\frac{3}{2} \frac{1}{\sqrt{3}}$ & $\frac{3}{2}\left(-\sqrt{\frac{2}{3}}\right)$ \\
$\rho \Xi$ & & & 2 & 0 & 0 \\
$\omega \Xi$ & & & & 0 & 0 \\
$\phi \Xi$ & & & & & 0
\end{tabular}


Table 12: Coefficients $C_{i j}^{I S}$ for the sector $I=3 / 2, S=-2$.

\begin{tabular}{l|cc} 
& $\bar{K}^{*} \Sigma$ & $\rho \Xi$ \\
\hline $\bar{K}^{*} \Sigma$ & -1 & -1 \\
$\rho \Xi$ & & -1
\end{tabular}

Table 13: Coefficients $C_{i j}^{I S}$ for the sector $I=0, S=-3$.

$$
\begin{array}{c|c} 
& \bar{K}^{*} \Xi \\
\hline \bar{K}^{*} \Xi & 0
\end{array}
$$

Table 14: Coefficients $C_{i j}^{I S}$ for the sector $I=1, S=-3$.

$$
\begin{array}{c|c} 
& \bar{K}^{*} \Xi \\
\hline \bar{K}^{*} \Xi & -2
\end{array}
$$

\section{References}

[1] N. Kaiser, P. B. Siegel and W. Weise, Phys. Lett. B 362, 23 (1995) [arXiv:nuclth/9507036].

[2] N. Kaiser, P. B. Siegel and W. Weise, Nucl. Phys. A 594, 325 (1995) [arXiv:nuclth/9505043].

[3] N. Kaiser, T. Waas and W. Weise, Nucl. Phys. A 612, 297 (1997) [arXiv:hep$\mathrm{ph} / 9607459]$.

[4] E. Oset and A. Ramos, Nucl. Phys. A 635 (1998) 99 .

[5] J. A. Oller and U. G. Meissner, Phys. Lett. B 500, 263 (2001) [arXiv:hep-ph/0011146].

[6] C. Garcia-Recio, M. F. M. Lutz and J. Nieves, Phys. Lett. B 582 (2004) 49.

[7] C. Garcia-Recio, J. Nieves, E. Ruiz Arriola and M. J. Vicente Vacas, Phys. Rev. D 67, 076009 (2003) [arXiv:hep-ph/0210311].

[8] T. Hyodo, S. I. Nam, D. Jido and A. Hosaka, Phys. Rev. C 68, 018201 (2003) [arXiv:nucl-th/0212026].

[9] T. Hyodo, D. Jido and A. Hosaka, Phys. Rev. D 75, 034002 (2007) [arXiv:hep$\mathrm{ph} / 0611004]$. 
[10] M. Doring, E. Oset and S. Sarkar, Phys. Rev. C 74, 065204 (2006) [arXiv:nuclth/0601027].

[11] V. K. Magas, E. Oset and A. Ramos, Phys. Rev. Lett. 95, 052301 (2005) [arXiv:hep$\mathrm{ph} / 0503043]$.

[12] D. Jido, E. Oset and T. Sekihara, arXiv:0904.3410 [nucl-th].

[13] E. E. Kolomeitsev and M. F. M. Lutz, Phys. Lett. B 585 (2004) 243 .

[14] S. Sarkar, E. Oset and M. J. Vicente Vacas, Nucl. Phys. A 750 (2005) 294 [Erratumibid. A $\mathbf{7 8 0}$ (2006) 78].

[15] D. Jido, M. Doering and E. Oset, Phys. Rev. C 77, 065207 (2008) [arXiv:0712.0038 [nucl-th]].

[16] T. Hyodo, D. Jido and A. Hosaka, Phys. Rev. C 78, 025203 (2008) [arXiv:0803.2550 [nucl-th]].

[17] A. Martinez Torres, K. P. Khemchandani and E. Oset, Phys. Rev. C 77, 042203 (2008) [arXiv:0706.2330 [nucl-th]].

[18] A. Martinez Torres, K. P. Khemchandani and E. Oset, Eur. Phys. J. A 35, 295 (2008) [arXiv:0805.3641 [nucl-th]].

[19] K. P. Khemchandani, A. Martinez Torres and E. Oset, Eur. Phys. J. A 37, 233 (2008) [arXiv:0804.4670 [nucl-th]].

[20] D. Jido and Y. Kanada-En'yo, Phys. Rev. C 78, 035203 (2008).

[21] Y. Kanada-En'yo and D. Jido, Phys. Rev. C 78, 025212 (2008) [arXiv:0804.3124 [nuclth]].

[22] P. Gonzalez, E. Oset and J. Vijande, Phys. Rev. C 79, 025209 (2009) [arXiv:0812.3368 [hep-ph]].

[23] S. Sarkar, B. X. Sun, E. Oset and M. J. V. Vacas, arXiv:0902.3150 [hep-ph].

[24] M. Bando, T. Kugo, S. Uehara, K. Yamawaki and T. Yanagida, Phys. Rev. Lett. 54, 1215 (1985).

[25] M. Bando, T. Kugo and K. Yamawaki, Phys. Rept. 164, 217 (1988).

[26] M. Harada and K. Yamawaki, Phys. Rept. 381, 1 (2003) [arXiv:hep-ph/0302103].

[27] U. G. Meissner, Phys. Rept. 161, 213 (1988).

[28] M. Doring, C. Hanhart, F. Huang, S. Krewald and U. G. Meissner, arXiv:0903.4337 [nucl-th]. 
[29] R. Molina, D. Nicmorus and E. Oset, Phys. Rev. D 78, 114018 (2008) [arXiv:0809.2233 [hep-ph]].

[30] H. Nagahiro, J. Yamagata-Sekihara, E. Oset and S. Hirenzaki, arXiv:0809.3717 [hep$\mathrm{ph}$.

[31] L. S. Geng and E. Oset, Phys. Rev. D 79, 074009 (2009) arXiv:0812.1199 [hep-ph].

[32] C. Garcia-Recio, J. Nieves and L. L. Salcedo, Phys. Rev. D 74, 034025 (2006) [arXiv:hep-ph/0505233].

[33] H. Nagahiro, L. Roca, A. Hosaka and E. Oset, Phys. Rev. D 79, 014015 (2009) [arXiv:0809.0943 [hep-ph]].

[34] Riazuddin and Fayyazuddin, Phys. Rev. 147, 1071 (1966).

[35] J.J. Sakurai, Currents and mesons (University of Chicago Press, Chicago Il 1969)

[36] F. Klingl, N. Kaiser and W. Weise, Nucl. Phys. A 624 (1997) 527 [arXiv:hep$\mathrm{ph} / 9704398]$.

[37] J. E. Palomar and E. Oset, Nucl. Phys. A 716, 169 (2003) [arXiv:nucl-th/0208013].

[38] G. Ecker, Prog. Part. Nucl. Phys. 35 (1995) 1

[39] V. Bernard, N. Kaiser and U. G. Meissner, Int. J. Mod. Phys. E4 (1995) 193

[40] A. Ramos, E. Oset and C. Bennhold, Phys. Rev. Lett. 89, 252001 (2002) [arXiv:nuclth/0204044].

[41] T. Inoue, E. Oset and M. J. Vicente Vacas, Phys. Rev. C 65, 035204 (2002) [arXiv:hep$\mathrm{ph} / 0110333]$.

[42] H. Nagahiro, L. Roca and E. Oset, Eur. Phys. J. A 36, 73 (2008) [arXiv:0802.0455 [hep-ph]].

[43] L. Roca, E. Oset and J. Singh, Phys. Rev. D 72, 014002 (2005) [arXiv:hep$\mathrm{ph} / 0503273]$.

[44] L. Tolos, A. Ramos and E. Oset, Phys. Rev. C 74, 015203 (2006) [arXiv:nuclth/0603033].

[45] M. Kirchbach and C. B. Compean, Eur. Phys. J. A 39, 1 (2007) arXiv:0805.2404 [hep-ph].

[46] C. Amsler et al. [Particle Data Group], Phys. Lett. B 667, 1 (2008). 
[47] C. Schutz, J. Haidenbauer, J. Speth and J. W. Durso, Phys. Rev. C 57, 1464 (1998); O. Krehl, C. Hanhart, S. Krewald and J. Speth, Phys. Rev. C 62, 025207 (2000); A. M. Gasparyan, J. Haidenbauer, C. Hanhart and J. Speth, Phys. Rev. C 68, 045207 (2003).

[48] M. Döring, private communication, in preparation.

[49] M. Doring, C. Hanhart, F. Huang, S. Krewald and U. G. Meissner, arXiv:0903.1781 [nucl-th].

[50] B. M. K. Nefkens, AIP Conf. Proc. 870, 405 (2006).

[51] J. W. Price et al. [CLAS Collaboration], Phys. Rev. C 71, 058201 (2005) [arXiv:nuclex/0409030]. 